optimiste que nous offrent les auteurs. Le plaidoyer est bien celui d'une météorologie des humeurs du couple Soleil-Terre. Les auteurs montrent qu'il y a les outils pour cela, comme la modélisation, l'intelligence artificielle, les centres de prévision, bien que des progrès restent à faire. Ils ont raison : il est souhaitable que la discipline se dote d'un système de prévision et d'information régulières des populations et des États, au même titre que la météorologie « habituelle ».

Pour conclure, ce livre est très bien construit. Les auteurs ne sont pas tombés dans le piège de la vulgarisation ni de la spécialisation. Ils ont réussi un bon équilibre entre les deux : chaque lecteur peut y trouver son compte. La bibliographie est en outre complète et il y a une sitographie internet bien utile permettant de continuer à s'évader. Le livre montre aussi de très belles photos. Pari réussi donc : félicitations aux auteurs et bonne lecture à tous !

Olivier Pujol

Météorologie de l'espace. Vivre demain avec notre soleil

Jean Lilensten, Frédéric Pitout, Marina Gruet, João Pedro Marques

de Boeck, 2021, 256 p, $25 €$

\title{
La petite histoire des flocons de neige par Étienne Ghys
}

V oici un livre agréable à lire à tout point de vue. L'auteur, mathématicien reconnu, raconte que cet ouvrage est né à la suite d'une conférence de mathématiques pour des enfants de l'école primaire. Le sujet est celui des flocons de neige. Il s'agit en fait de cristaux de glace, dont on comprend le lien avec les mathématiques en raison de leur symétrie, et non de flocons de neige, qui sont des associations de cristaux donnant des structures plus désordonnées.

L'ouvrage possède plusieurs degrés de lecture. L'auteur choisit de nous raconter la science des cristaux sur environ cinq siècles à partir d'une vingtaine de portraits variés : un archevêque catholique, un philosophe, des physiciens, un pêcheur, une lady, un médecin, un photographe amateur et, bien sûr, des mathématiciens. L'auteur montre comment tous ces protagonistes se sont interrogés au sujet de la neige et ont permis à ce que nous la comprenions de mieux en mieux. On retiendra la contribution remarquable de Kepler, bien connu en astronomie, pour avoir, le premier, souligné la symétrie des cristaux malgré leur diversité et posé la question fondamentale de leur planéité. Chaque portrait est agrémenté de nombreux dessins, de photos, de commentaires et d'anecdotes qui rendent la lecture agréable. Un petit bémol dans cette liste de portraits est la parenthèse sur la diffraction. Elle est un peu longue et son absence n'aurait pas gêné la

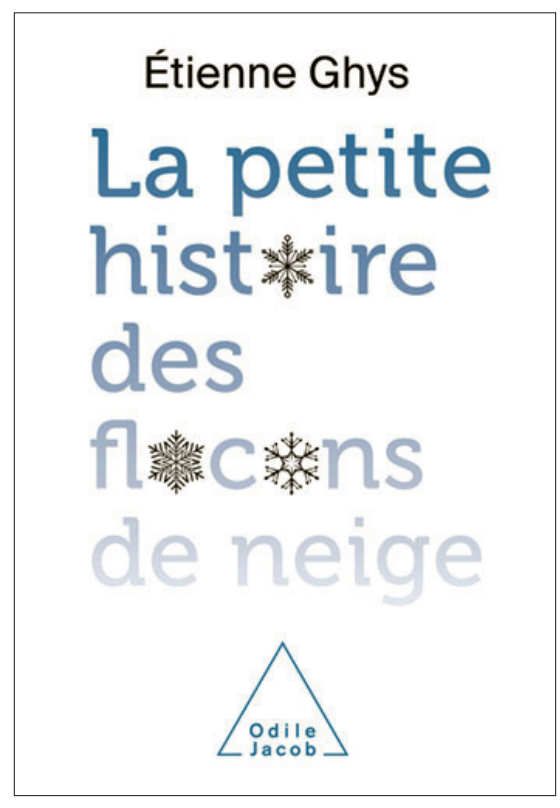

portée de cet ouvrage. N'oublions pas les magnifiques planches de Nakaya et sa classification des formes de cristaux selon la température et l'humidité. Il est dommage que l'auteur ne soit pas allé un peu plus loin, car la classification de Nakaya a dû être revue : elle est trop parfaite. Le diagramme de Bailey et Hallett ${ }^{1}$ est plus réaliste. Même si certains éléments du tableau de Nakaya sont conservés, on sait désormais que les cristaux irréguliers et imparfaits sont dominants.

1. Bailey M.P., Hallett J., 2009. A comprehensive habit diagram for atmospheric ice crystals: Confirmation from the Laboratory, AIRS II, and other field studies. J. Atm. Sci., 66, 2888 2899.
La fin de l'ouvrage porte, mathématiquement, sur les symétries et la croissance cristalline. On apprend pourquoi les cristaux ont une symétrie sous-jacente hexagonale et non pas pentagonale, et on fait connaissance avec quelques règles mathématiques simples qui rendent compte de la croissance des cristaux. L'auteur présente ces modèles mathématiques comme un jeu. Cela ravira les enfants, certes, et les spécialistes pourront y trouver une source d'inspiration. Le dernier portrait est celui d'un artiste, rappelant à juste titre que l'art est un vecteur de connaissances scientifiques et, parfois, un catalyseur de l'imagination.

Pour conclure, ce petit livre est une réussite. Soulignons l'effort fait pour agrémenter le texte de magnifiques photos, de dessins et de recommandations de sites internet pour continuer l'exploration. La bibliographie est, elle, limitée à une section "Crédits des illustrations » ; dommage, mais on peut comprendre que l'auteur, de par son objectif, n'ait pas souhaité inonder son discours de références à des articles trop ardus. Quoi qu'il en soit, ce livre fera passer un bon moment à tous.

Olivier Pujol

La petite histoire des flocons de neige Étienne Ghys Odile Jacob, 2021, 144 p., 16,90 €. 